\title{
Modified newtonian dynamics: success and problems on globular cluster scale
}

\author{
HongSheng Zhao $\dagger$ \\ University of St Andrews, School of Physics and Astronomy, KY16 9SS, UK \\ National Astronomical Observatory of China, Chinese Academy of Science, Datun Road A20, \\ Chaoyang District, Beijing, 100012, China
}

\begin{abstract}
Many past attempts to kill MOND have only strengthened the theory. Better data on galaxy velocity curves clearly favor MOND (without fine-tuning) over cold dark matter. The usual critism on the incompleteness of classical MOND has spurred a Modified Relativity (MR) by Bekenstein. After outlining cosmology and lensing in MOND, we review MOND on small scales. We point out some potential problems of MOND in two-body relaxation and tidal truncation. We argue that the tidal field in any MOND-like gravity theory predicts that the Roche lobe sizes of a binary system are simply proportional to the binary baryonic mass ratio to the power $1 / 3$. An immediate application of this result is that the tidal field and tidal truncation radii of million-star globular clusters and million-star dwarf galaxies (e.g., the Milky Way satellites NGC2419 and Carina) would be very similar because of the one-to-one relation between gravity and baryon distribution. This prediction appears, however, inconsistent with the fact that all globulars are truncated to much smaller sizes than all dwarf galaxies. Whether tide is uniquely determined by baryons can also be used to falsify any MOND-like gravity theory, whether classical or relativistic.
\end{abstract}

Keywords. dark matter

\section{Introduction}

A large amount of astronomical data suggests that the $G M / r^{2}$ Newtonian gravity from the baryonic material in galaxies fails to explain the large accelerations implied by motions in spiral and elliptical galaxies. However, Zwicky's proposal to introduce unseen gravitating (dark) mass with a very flexible density distribution wherever needed for the motions of galaxies and stars has been troubled by a lack of experimental confirmation of its elementary particle counterparts for more than 60 years. Evidence is also summarized in recent articles about the difficulties facing dark matter (DM) theories on galaxy scales (Spergel \& Steinhardt 2000, Ostriker \& Steinhardt 2003). Surprisingly, an alternative gravity theory with Modified Newtonian Dynamics (MOND, Milgrom 1983) has been doing very well exactly where the dark matter theory is doing poorly. The predictive power of this 20-year-old classical theory with virtually no free parameters (Bekenstein \& Milgrom 1984) is recently highlighted by the astonishingly good fits to contemporary kinematic data of a wide variety of high and low surface brightness spiral and elliptical galaxies; even the fine details of the ups and downs of velocity curves are elegently reproduced without fine tuning of the baryonic model (Sanders \& McGaugh 2002, Milgrom \& Sanders 2003). Originally it was proposed that galaxy rotation curves could be fit by motions in a modified gravity $\mathbf{g}$, which is significantly stronger than Newtonian gravity

$\dagger$ Overseas Outstanding Young Scholar in Beijing Observatory and PPARC Advanced Fellow 
in the weak regime defined by a gravitational field energy density

$$
\frac{|\mathrm{g}|^{2}}{8 \pi G} \leqslant \frac{a_{0}^{2}}{8 \pi G} \approx \rho_{b}(0) c^{2}
$$

where $G$ is the gravitational constant, and $\rho_{b}(0) c^{2}$ is the present universal mean baryon energy density (commonly the weak regime is defined as where gravity $g \leqslant a_{0} \sim 1.2 \times$ $10^{-8} \mathrm{~cm} \mathrm{sec}^{-2}$ ). Far away from the baryons, the modified gravity $\mathbf{g}$ satisfies

$$
\frac{\mathbf{g}}{\tilde{G}(g)} \approx \frac{\mathbf{g}_{N}}{G} \equiv-\sum_{i} \frac{M_{i} \hat{\mathbf{R}}_{i}}{\left|R_{i}\right|^{2}}
$$

where $\mathbf{g}_{N}$ is the vector sum of Newtonian gravity (distance-inverse-squared attractive force) of all baryonic particles with masses $M_{i}$ at distances $R_{i}$, where the effective gravitational constant $\tilde{G}$ can be chosen as, e.g., $\frac{G}{\tilde{G}(g)}=1-\exp \left[-\left(\frac{g}{a_{0}}\right)^{\alpha}\right]$, where $\alpha=1$ gives the MOND gravity $\mathrm{g}$. We get a MOND-like theory for any positive $\alpha$. In these theories, the only matter that matters is the luminous (baryonic) matter, and the gravitational field is a unique function of the luminous matter distribution.

These alternative theories based on modifying the law of gravity in a purely baryonic universe have been gaining ground rapidly. Milgrom's empirical formula is a simplified treatment of a classical theory with a curl-free gravitational field by Bekenstein \& Milgrom (1984), which is now extendable to a Modified Relativistic (MR) theory (Bekenstein 2004) which passes standard and cosmological tests used to check General Relativity (GR). This recent breakthrough by Bekenstein transforms the empirical MOND formula to a falsifiable theory with respectable theoretical underpinnings comparable almost to the rival dark matter theory. Here we examine various aspects of the MOND theory after outlining cosmology and lensing in the relativistic MOND.

\subsection{Cosmology and Lensing}

Bekenstein (2004) has shown that MONDian cosmology and lensing follow the standard GR formulation. The physical metric of the FRW universe in MOND is written as,

$$
g_{\mu \nu} d x^{\mu} d x^{\nu}=c^{2} d t^{2}-R(t)^{2}\left[d \chi^{2}+f(\chi)^{2}\left(d \theta^{2}+\sin ^{2} \theta d \phi^{2}\right)\right]
$$

where $f(\chi)=\sin \chi, \chi, \sinh \chi$ for closed, flat and open universe as in FRW GR, and the physical scale factor is $R(t)=R_{0} /(1+z)$. Note that we have removed the tilde sign that Bekenstein uses to denote physical coordinates. if we introduce the vaccum energy and neglect the scale field $\phi$ as in Bekenstein, then the MONDian universe expands with a Hubble parameter $H(z(t))=\frac{d \ln R(t)}{d t}$ given approximately by

$$
\frac{H^{2}(z)}{H_{0}^{2}} \approx \Omega_{m}(1+z)^{3}+\Omega_{r}(1+z)^{4}+\Omega_{K}(1+z)^{2}+\Omega_{\Lambda}, \Omega_{K} \equiv 1-\Omega_{m}-\Omega_{r}-\Omega_{\Lambda},
$$

where $\Omega_{r} \sim 10^{-4} h^{-2}$ is normalised by the $2.7 \mathrm{~K} \mathrm{CMB}$, and $\Omega_{m} \sim 0.05 h^{-2}$ from all observable baryons, and $\Omega_{K}$ is the curvature term. The matter-radiation equality happens at redshift of about $\Omega_{m} / \Omega_{r} \sim 1000$; this is much lower than in CDM models.

To do lensing, Bekenstein shows (in eq. 116) that for small perturbations of the metric by a potential $\Phi$, light travels on geodesic given by

$$
d s^{2}=\tilde{g}_{\mu \nu} d x^{\mu} d x^{\nu} \approx-\left(1+\frac{2 \Phi}{c^{2}}\right) c^{2} d t^{2}+\left(1-\frac{2 \Phi}{c^{2}}\right) d l^{2}=0
$$


where $d l$ is the proper length. Hence the light travel time

$$
d t \approx\left(1-\frac{2 \Phi}{c^{2}}\right) \frac{d l}{c},
$$

same as in Einstein universe. The gravititional potential satisfies approximately the MOND eq.

$$
-\nabla \cdot\left[\mu\left(\frac{g}{a_{0}}\right) \mathbf{g}\right]=4 \pi G \rho, \quad \mathbf{g}=-\nabla \Phi,
$$

where $\rho$ is the gravitational mass density of baryonic matter, and the $\mu$ is the usual dimensionless function of the rescaled gravity $\frac{g}{a_{0}}$ introduced by Milgrom.

The deflection for a light array of the closest approach (roughly impact parmameter, physical length) $b$ is calculated by

$$
\alpha=\int \frac{2 g_{\perp} d t}{c}
$$

where $g_{\perp}=g(r) \cdot \frac{b}{r}$ is the perpendicular component of the gravity $g(r)$ at radius $r=$ $\sqrt{(c t)^{2}+b^{2}}$, and $c t$ is the path length along the line of sight counted from the point of closest approach. Hence the deflection angle

$$
\alpha=\int_{-\infty}^{\infty} \frac{2 g(r) d t}{c} \frac{b}{\sqrt{(c t)^{2}+b^{2}}} .
$$

This is essentially the same as eq. (109) of Bekenstein.

\section{Two-body Relaxation Time in MOND}

As pointed by Ciotti \& Binney (2004), relaxation in MOND is a non-trivial calculation because it is a long-range gravity with a divergent potential at large distance. However, by and large, MOND gravity is effectively a stronger-than-Newtonian gravity. This suggests that we can rescale Newtonian gravity by a factor $\frac{\tilde{G}}{G}$. To balance this stronger gravity, a bound object of certain size $r$ acquires a higher internal velocity (than Newtonian case with pure baryons), hence shorter dynamical time, and faster relaxation since the relaxation time is proportional to dynamical time with

$$
t_{x} \propto N t_{\mathrm{dyn}} \sim \frac{N r}{C V}
$$

where $C \sim 7 \ln N \sim 50$ is a large constant related to the Coulomb logarithm, and the dynamical time

$$
t_{\text {dyn }}=\left(\frac{r}{V}\right) \sim 1 \mathrm{Myr} \frac{r}{\mathrm{pc}} \frac{1 \mathrm{~km} \mathrm{~s}^{-1}}{V}
$$

where $r$ is the half-mass radius, and $V$ is characteristic circular velocity. So for $N \sim 10^{4-5}$ globular cluster, it relaxs on time scales of 100-1000 dynamical times. Typical dynamical time is of order Myr, hence a globular is generally relaxed in a fraction of the Hubble time. In MOND the circular velocity is generally given by

$$
V=\left(G M a_{0}\right)^{1 / 4}=3.5 \mathrm{~km} \mathrm{~s}^{-1}\left(\frac{M}{10^{4} M_{\odot}}\right)^{1 / 4}
$$

if in deep-MOND where $r \geqslant \sqrt{\frac{G M}{a_{0}}}=3.5 \mathrm{pc} \sqrt{\frac{M}{10^{4} M_{\odot}}}$. 


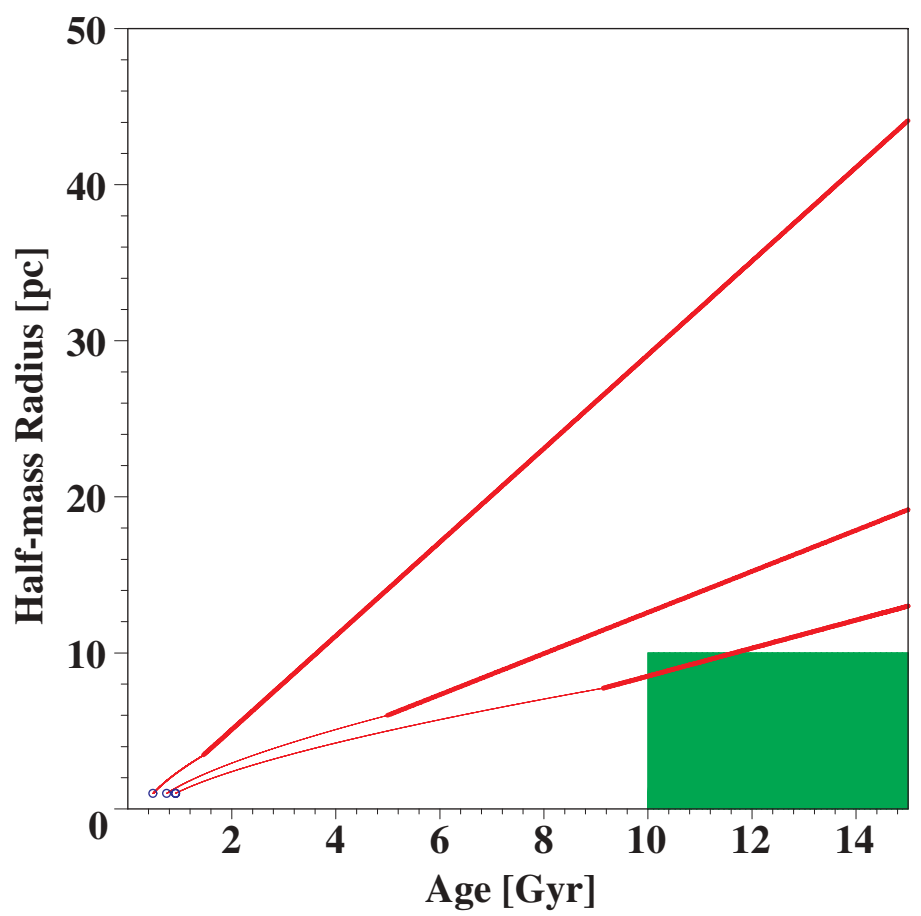

Figure 1. shows the relaxation-driven expansion of the half-mass radius of an isolated globular cluster of $N=2 \times 10^{4}, 6 \times 10^{4}, 1 \times 10^{5}$ (curves from top to bottom) half-solar-mass stars with a pre-core-collapse size of $1 \mathrm{pc}$ (marked by the blue circles). The change from strong to weak gravity is indicated by the change from thin lines to thick lines. Observed globulars typically occupy the green shaded zone in the age vs. size plane.

Relaxation drives a globular to expand. Star clusters might have been born very compact, and in the strong-gravity regime. However, fast relaxation means that globular clusters cannot stay small, will eventually expand and enter the weak regime. The relaxation time can be expressed in terms of some kind of rate of expansion per relaxation time

$$
\frac{r}{t_{x}}=\frac{50 \mathrm{pc}}{10 \mathrm{Gyr}} \frac{10^{4}}{N}\left(\frac{V}{1 \mathrm{~km} \mathrm{~s}^{-1}}\right), \quad V=3.5 \mathrm{~km} \mathrm{~s}^{-1}\left(\frac{M}{10^{4} M_{\odot}}\right)^{1 / 4},
$$

where we have assumed $C \sim 7 \ln N \sim 50$, and have used the circular velocity in the MOND regime. The above implies that a cluster should expand to a size of $\sim 175 \mathrm{pc}$ over a Hubble time in MOND.

To illustrate this, we give a few examples in Fig. 1. Consider, for example, a hypothetical cluster born fully isolated with $N=2 \times 10^{4}$ half-solar-mass stars inside a half-mass radius of $1 \mathrm{pc}$. The cluster is initially dense and in the strong gravity regime. Rapid relaxation leads to core collapse after about $t_{0}=0.4$ Gyrs where we take $t_{0}$ as 10 times the initial relaxation time $t_{x}$. Afterwards the cluster expands as $t^{2 / 3}$ in strong gravity till $r_{h}$ reaches about $3 \mathrm{pc}$ at about $t=1.4 \mathrm{Gyr}$, after which the cluster enters the weak gravity regime, $r_{h}$ expands linearly with time according to MOND, and reaches a size $r_{h}=45 \mathrm{pc}$ after a Hubble time. Systems with larger N grows slower (Fig. 1). Nevertheless, clusters with $N=2 \times 10^{4}$ to $1 \times 10^{5}$ half-solar-mass stars all grow to a half-mass size $12-45 \mathrm{pc}$, too big to be consistent with observed globular clusters. 


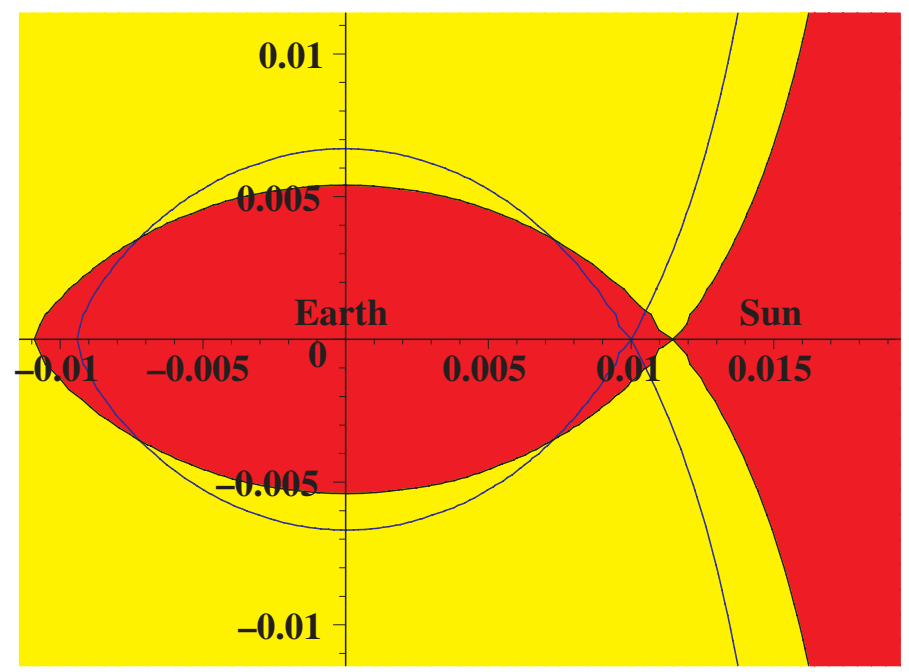

Figure 2. shows the rescaled Roche lobes (contours of the effective potential) in the equatorial plane of a hypothetical isolated Earth-Sun binary mass ratio $3 \times 10^{-6}$ in the strong gravity regime (say with the separation $1 \mathrm{AU}$, thin blue lines), and in the weak gravity regime (say with separation $1 \mathrm{pc}$, and $G M_{\odot} / 1 \mathrm{pc}^{2} \sim 0.001 a_{0} \ll a_{0}$, shaded areas). The Earth is at origin and the Sun is at rescaled unit length to the right. The inner Lagrangian point is a saddle point between the Earth and the Sun, which is slightly further away from the Earth in the deep-MOND regime than in strong gravity regime.

\section{Tidal truncations or Roche lobes of binary systems}

First we note that at large distances from the baryonic particles Gauss's theorem predicts that a MOND-like gravity $\mathbf{g}$ with an effective gravitational constant $\tilde{G}(g)$ is approximately parallel to the direction of the Newtonian gravity $\mathbf{g}_{N}$ with the amplitude satisfying eq. (1), so the gravity $\mathbf{g}$ is determined by the total mass of the baryons $\sum_{i} M_{i}$ and the mean distance $r$, independent of the spatial distribution of the baryons. This is true even in the rigorous classical counterpart (Bekenstein \& Milgrom 1984) of the Bekenstein theory. So, e.g., a dense globular cluster and a fluffy dwarf galaxy of $10^{5} L_{\odot}$ should have comparable baryonic mass, hence the similar gravity field on scales of kpc, nearly independent of the internal density profile. Indeed MOND nicely accounts for the very small scatter of dynamical properties of structures of similar baryonic distribution on galaxy scale (Sanders McGaugh 2002, McGaugh 2000, 2004). However, this "nice feature" of baryonic gravity also means that the dynamics of wide binary stars would be a scaled-down version of the Antenna-like merging galaxies.

Theoretically in a binary system, the Roche radius (or tidal radius or virial radius when referring to galaxies sometimes) is where the average density of the mass-losing satellite is comparable to the average density of the mass-receiving host at some point $R$ along the orbit of satellite, i.e.,

$$
\frac{G m}{\frac{4 \pi}{3} r_{t}^{3}} \approx k \frac{G M}{\frac{4 \pi}{3} R^{3}},
$$

with the prefactor $k$ ranging from 1 to 3 in different definitions in the literature (Binney \& Tremaine 1987); e.g., $k=2$ according to von Hoerner (1957). This well-known tidal criterion in Newtonian gravity is in fact a result of basic dynamics, independent of the force law parameter $\tilde{G}(g)$ and applies to any gravity. It is shown elsewhere (Zhao et al. $2005)$ that the prefactor $k=2$ rigourously in the deep-MOND regime. The shape of the 


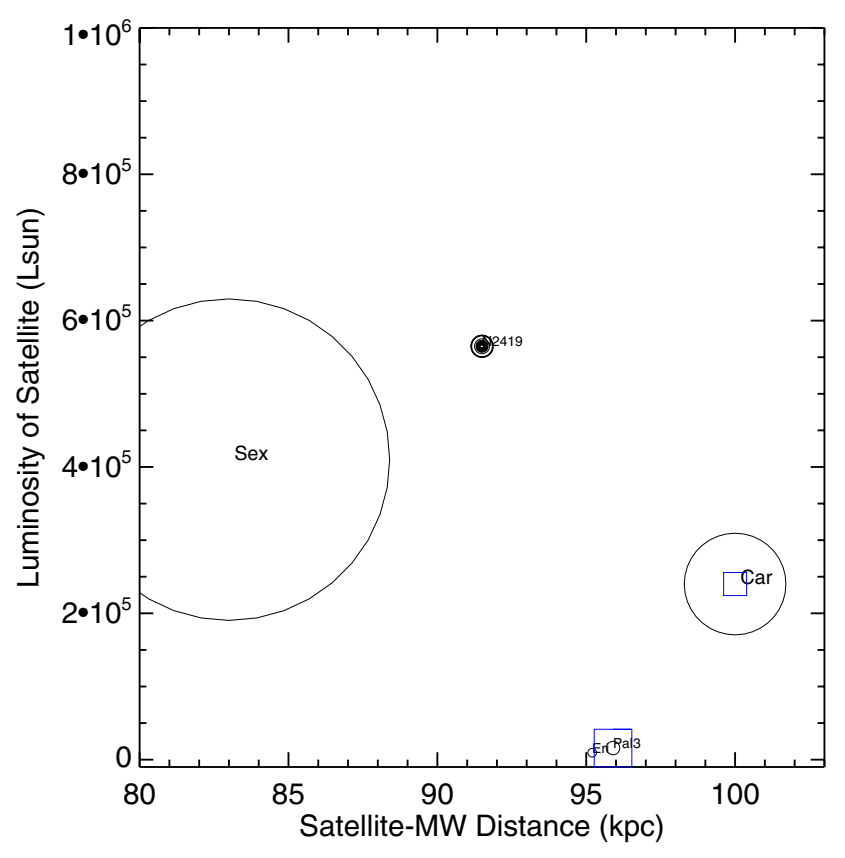

Figure 3. shows the satellite distance vs. luminosity scatter diagram for the dwarf galaxies (labeled with larger font) and globular clusters (labeled with smaller font) orbiting in Milky Way's very outer halo (with orbital periods about one quarter of a Hubble time). The sizes of the symbols are scaled to the physical sizes of the satellites (as a reference, the faint Eridanius cluster at the bottom is 100pc, and Sextans dwarf is 3000pc). The sizes of all globulars of the Milky Way are all drawn as smaller rings inside NGC2419, which is the largest, and most distant bright globular cluster. Predicted sizes of the faint Pal3 cluster and Carina dwarf are also shown as squares based on their proper motion inferred pericentres in a $10^{11} L_{\odot}$ baryonic Milky Way.

Roche lobes is somewhat more squashed than the Newtonian counterpart. An example of Roche lobes is given in Fig. 2 .

\subsection{Application to satellite globular clusters and dwarf galaxies}

The Roche criteria (eq. 3.1) predicts that objects should have same sizes if they have similar baryonic content in similar enviornment. This, however, is inconsistent with basic observation data, and we see a large scatter for the truncation radii of outer globular and a dozen dwarf galaxy satellites accelerating in the tide and very weak gravity of a luminous host galaxy, like the Milky Way (see Fig. 3). So the observed limiting radii of these satellites (Harris 1996, Mateo 1998) are no longer simply and uniquely scaled with the binary baryonic mass ratio as predicted by a MOND-like theory (eq. 3.1).

For example, NGC 2419, the largest cluster of the Milky Way, is as distant as and 1-2 times as luminous as the $(2.5-4) \times 10^{5} L_{\odot}$ dwarfs Carina/Sextans, which has an equilibrium of stars extending to $1000-3000 \mathrm{pc}$ radii at a distance of about $90 \mathrm{kpc}$ from the Milky Way. A baryonic universe predicts that NGC 2419 should resist the tide of the Milky Way as well as the Sextans dwarf does on 3000pc scale; Sextans shows no sign of being disrupted (Grebel et al. 2000). Nevertheless, even this largest globular is truncated on 200pc scale. Such large discrepancy does not go away if we take into account of different orbits or mass of the satellites, e.g., a baryonic universe would predict that the cluster Pal3 (faint $10^{4} L_{\odot}$ and presently near pericentre at $90 \mathrm{kpc}$, Dinescu et al. 1999) 
is bigger than the Carina dwarf (presently near apocentre, Piatek et al. 2003), but in reality as shown in Fig. 3, Pal3 (Sohn et al. 2003) is much smaller than Carina (Walcher et al. 2003). Note that orbits in MOND is not much different from orbits in isothermal halo potentials (Read \& Moore 2005).

This example points out a generic problem general to galaxies with resolved satellite globulars vs. dwarfs in any baryonic universe including Bekenstein's cosmology: why there is a clear size gap between $250 \mathrm{pc}-400 \mathrm{pc}$ and nature forms neither very big globulars nor very small dwarfs despite a good mix of the baryonic mass and enviornment variables (orbital phase and pericentric tide) of these satellites. In a baryonic universe "globulars" and "dwarf ellipticals" are practically similar assembly of stars described by the King models: they are pressure-supported low-dispersion gas-poor low-luminosity elliptical equilibria immersed in and sculptured by the tidal field of a common host galaxy.

\subsection{Application: the sizes of the globular clusters in Fornax}

Could it be that the globulars were born small and stayed small? It is plausible that globulars might have formed from denser and cooler gas clouds than stars in some dwarfs. However, a very dense core of a globular is theoretically unstable to stellar encounters and must evolve rapidly, and on a Hubble time typically 10-100 percent of the initial mass of a star cluster are unbound due to relaxation-driven stellar evaporation (Johnstone 1993). Relaxation also drives the half-light radius of a globular to expand as a power law of the time $t^{2 / 3}$ if we assume globulars are born infinitely compact and dense so we can neglect the delay for the core to collapse. Using eq. (1) and Fig. 1 of the numerical simulations of (Giersz \& Heggie 1996), we estimate the radius containing 90 percent of the mass of a typical globular cluster of $10^{5}$ half-solar mass stars expands as $100 \mathrm{pc}\left(\frac{t}{10 \mathrm{Gyr}}\right)^{2 / 3}$.

Relaxation is even faster in MOND (Ciotti \& Binney 2004) because of the stronger gravity, higher velocity dispersion and faster dynamical time (Baumgardt et al. 2005). A detailed calculation of the expansion rate in MOND is given elsewhere (Zhao et al. 2005). Essentially a globular cluster born very compact in MOND cannot stay compact.

There are five bright old globular clusters orbiting around the Fornax dwarf elliptical, $140 \mathrm{kpc}$ away from the Milky Way centre. These globulars have a typical baryonic mass of $10^{4-5} M_{\odot}$ enclosed in a limiting radius of 30-50 pc with a half-mass radius of 4-12 pc (using data compiled in Mackey \& Gilmore 2003 and Rodgers \& Roberts 1994). One of clusters, No. 1, has a distorted profile and appears to be tidally disturbed. The clusters No. 3,4,5 appear to have excess stars near their limiting radii. The clusters No. 1,2,5 are on the outskirts of Fornax, between 1-1.5kpc from the Fornax centre. Our model of relaxation-driven expansion predicts (cf. Fig. 1) that Fornax No. $1\left(1 \times 10^{4} L_{\odot}\right)$ should have grown to a half-mass size of about $40 \mathrm{pc}$, and more massive clusters No. 2 and No. 5 $\left(5 \times 10^{4} L_{\odot}\right)$ should grow to $12 \mathrm{pc}$. The predicted sizes are significantly greater than the observed half-light radius of $12 \mathrm{pc}$ (No.1) and 6pc (No. 2 and No. 5).

Our model also predicts (cf. eq. 3.1) that the Roche lobe of these Fornax clusters should have a radius of $(650-1000)$ pc if they are on a $140 \mathrm{kpc}$ orbit around the Milky Way $\left(5 \times 10^{10} L_{\odot}\right)$, or a Roche lobe radius of $(100-130)$ pc if they are on a $1.5 \mathrm{kpc}$ orbit around Fornax $\left(2 \times 10^{7} L_{\odot}\right)$. Both of these predictions are much greater than the observed limiting radius of $(40-50)$ pc for all Fornax clusters.

\subsection{Discussion}

The limiting size to which a globular can maintain internal equilibrium are most likely limitted by the tidal force of the Milky Way in general. This is supported by the observation of a characteristic sudden change of outer density profile of most globulars and transient non-spherical outer features, as illustrated by the beautiful stream of Pal 5 found 


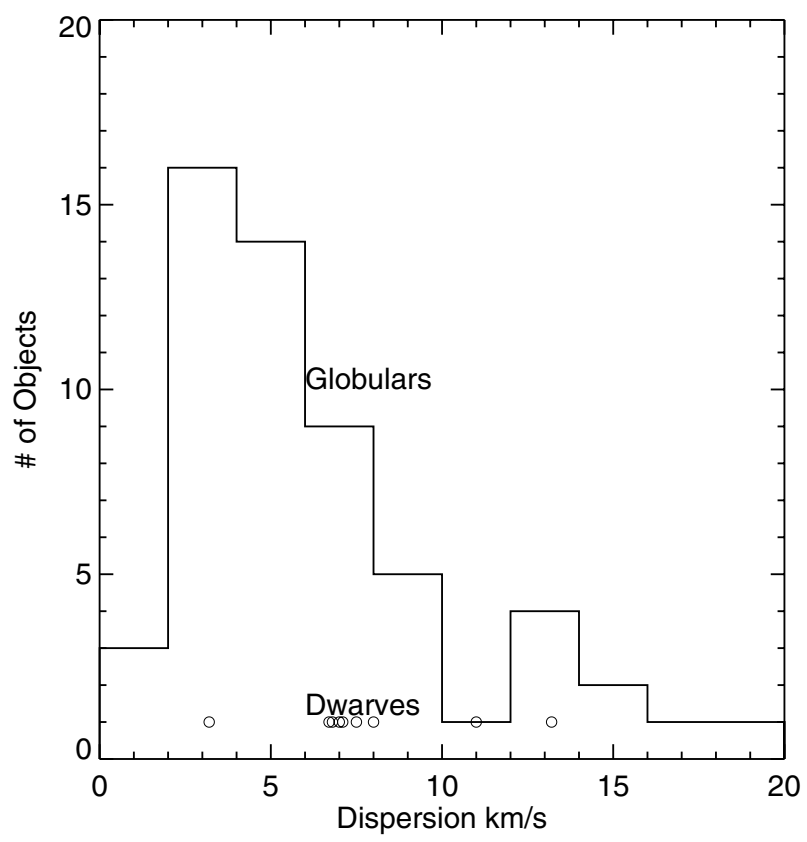

Figure 4. shows the central line of sight velocity dispersion of Galactic globular clusters (in histogram), and the velocity dispersion of Galactic dwarf galaxies (circles). Data taken from Gnedin et al. (2002) and Mateo (1998).

by Sloan Digital Sky Survey, and many distant clusters, e.g., NGC5694 and NGC1904, when deep observations have been taken along lines of sight with least background confusion (Leon et al. 2000, Odenkirchen et al. 2003, Grillmair et al. 1995,1996, Lehmann \& Scholz 1997). These features are most likely due to stars leaking from a boundary set by the Roche lobe much like the donor star in a binary evolving off the main sequence and transfering mass to its companion. The MOND gravity has a $\ln (r)$ infinite potential well, and so if in isolation a globular will grow indefinitely due to two-body relaxation. So both two-body relaxation and violent relaxation tend to smooth out any sharp initial boundaries. It is hard to imagine a non-tidal mechanism to sustain sudden changes of the density profiles. A globular must have a finite potential well or an outer truncation also because large amount of recycled gas from aging and evolved stars must flow out of the tidal radii of a globular as old globulars keep less than $1 M_{\odot}$ gas inside (Spergel 1991).

The cores of many Galactic globulars are as hot as dwarf galaxies with central velocity dispersion $\geqslant 8 \mathrm{~km} / \mathrm{s}$ (cf. Fig. 4), and some dwarf galaxies, e.g., Sextans (Kleyna et al. 2004) are colder than most globulars. An isolated object enters weak regime if bigger than $\sqrt{G M / a_{0}} \sim 3.5 \mathrm{pc} \sqrt{M / 10^{4} M_{\odot}}$, hence dwarf galaxies, and outer globulars like NGC2419 and Palomar clusters are all partly or entirely in the weak gravity regime. Nature sometimes put star clusters inside a small galaxy (as in Fornax, WLM and Sagittarius dwarf galaxies), but never vice versa. In fact there are evidences that some globulars are stripped out from the envelopes or carved from the cores of dwarf galaxies by galactic tide, which explains the similar orbits and smooth transition of baryonic mass of these two populations of satellites. It seems that while MOND-like gravity theories can account rotation curves of galaxy-scale structures without fine tuning, they do not genericly come with 
an important ingredient or sub-galaxy scale parameter that distinguishes dwarfs from globulars. Having dark matter in dwarves but not in globulars is perhaps one way out of the dilemma of a baryonic universe.

\section{Acknowledgements}

This proceeding was partly written during an extended visit to Beijing Observatory, funded through the Overseas Outstanding Young Scholarship program of the Chinese National Science Fundation. I would like to thank the Beijing Observatory for hospitality. I also acknowledge discussions with David Bacon, Xuelei Chen, Douglas Heggie, Keith Horne and Andy Taylor.

\section{References}

Baumgardt H., Grebel E.K. \& Kroupa P. 2004, MNRAS (astro-ph/0501272)

Bekenstein J. 2004, PhRvD, 70, 3509 (astro-ph/0403694)

Bekenstein J. \& Milgrom M. 1984, ApJ 286, 7

Binney J. \& Tremaine S. 1987, Galaxy dynamics, Princeton University Press.

Ciotti L. \& Binney, J. 2004, MNRAS 351, 285

Dinescu, D.I., Girard, T.M. \& van Altena, W.F. 1999, AJ 117, 1792

Grebel E.K., Odenkirchen M. \& Harbeck, D. 2000, AAS 200, 4607

Grillmair C.J., Ajhar E.A., Faber S.M., et al. 1996, AJ 111, 2293,

Harris, W.E. 1996, AJ 112, 1487

Giersz M. \& Heggie D.C. 1996, MNRAS 279, 1037

Gnedin O., Zhao H., et al. 2002, ApJ 568, L23

Johnstone, D. 1993, AJ 105, 155,

Kleyna J., Wilkinson M., Evans W. \& Gilmore G. 2004, MNRAS 354, L66

Lehmann I. \& Scholz R.D. 1997, A\&A 320, 776

Leon S., Meylan G. \& Combes F. 2000, $A \& A A 359,907$

Mateo M. 1998, ARA\&A 36, 435

McGaugh, S., et al. 2000, ApJ 553, 99

McGaugh S. 2004, ApJ 609, 652

Milgrom M. 1983, ApJ 270, 365

Milgrom M. \& Sanders R. H. 2003, ApJ 599, L25

Odenkirchen M. et al. 2003, AJ 126, 2385

Ostriker J. \& Steinhardt P. 2003, Science 300, 1909

Piatek S. et al. 2003, AJ 126, 2346

Read J. \& Moore B., 2005, astro-ph/0501273

Spergel D.N. 1991, Nature 352, 221

Spergel D.N. \& Steinhardt P. 2000, PRL 84, 3760

Sanders, R. \& McGaugh S. 2002, ARA\&A 40, 263

Sohn Y. et al. 2003, AJ 126, 803

von Hoerner, S. 1957, ApJ 125, 451

Walcher C.J., Fried J.W., Burkert A. \& Klessen R.S. 2003, A\& A 406, 847

Zhao H.S., et al. 2005, in preparation. 